\title{
EVALUACIÓN DE LAS MEDIDAS DE GRUPA Y MUSLO DE LA CRÍA Y UBRE DE LA MADRE COMO INDICADORES FENOTÍPICOS EN LA SELECCIÓN TEMPRANA DE LLAMAS (Lama glama) PARA CARNE
}

\author{
EVALUATION OF HIP AND THIGH MEASUREMENTS OF THE LAMA CRIA AND THE SIZE OF THEIR \\ DAM'S UDDERS AS PHENOTYPIC INDICATORS FOR THEEARLY SELECTION OF LAMAS (LAMA \\ GLAMA) FOR MEAT PRODUCTION
}

\author{
Otto Zea M. ${ }^{1}$, Víctor Leyva V. ${ }^{2}$, Wilber García V. ${ }^{3}$ y Néstor Falcón P. ${ }^{4}$
}

\section{Resumen}

Se utilizó un rebaño de 82 llamas con sus respectivas crías para evaluar el área de la grupa y el volumen del muslo de la cría, y el tamaño de la glándula mamaria de las madres como posibles indicadores para la selección temprana de llamas para producción de carne. En las crías se evaluó el desarrollo del área de la grupa y el volumen del muslo a los 2, 8 y 15 meses de edad, así como la correlación de estas medidas con el peso corporal y el perímetro torácico. En las madres se evaluó la relación entre el tamaño de la ubre y el peso de la cría al destete. El tamaño de la grupa se calculó como una figura geométrica y su área se determinó con el software Autocad, y el tamaño del muslo se expresó como el volumen de un cono truncado. El incremento porcentual desde los 2 hasta los 15 meses de edad fue significativamente mayor $(\mathrm{p}<0.05)$ en el peso corporal $(65.3 \%)$, área de la grupa $(51.5 \%)$ y volumen del muslo $(53.2 \%)$ que en el perímetro torácico $(32.9 \%)$. Las diferencias establecidas en la clasificación por tamaño de la grupa a los dos meses de edad se mantuvieron en las mediciones realizadas a los 8 y 15 meses de edad ( $<<0.05)$, y estas diferencias se mantuvieron en las otras variables bajo estudio (volumen de muslo, perímetro torácico y peso corporal), lo cual indica que la tasa de incremento fue similar en ambos grupos. Los coeficientes de correlación entre el área de la grupa, volumen del muslo, peso corporal, perímetro torácico y edad desde los 2 a los 15 meses de edad fueron altos ( $\mathrm{p}$ 0.001). Las correlaciones entre las medidas de la ubre de las madres y el peso corporal de las crías al destete fueron bajas, excepto para la relación de distancia entre pezones anteriores con peso de la cría al destete (0.46). Los resultados sugieren que el área de la grupa y volumen del muslo pueden ser usados como indicadores genéticos para la selección temprana de crías de llama para producción de carne por mantener una correlación alta y significativa con el peso corporal y el perímetro torácico.

Palabras clave: grupa, muslo, glándula mamaria, indicadores fenotípicos, tasa de crecimiento

\footnotetext{
${ }^{1}$ Práctica privada

${ }^{2}$ Laboratorio de Reproducción Animal,FMV-UNMSM.E-mail: vleyva@vet.unmsm.edu.pe

${ }^{3}$ Estación Experimental del Centro de Investigaciones IVITA-La Raya, FMV-UNMSM

${ }^{4}$ Laboratorio de Medicina Veterinaria Preventiva, FMV-UNMSM
} 


\section{Abstract}

A flock of 82 llamas with their calves was used to evaluate the hip area and thigh volume of the young and the size of the mammary gland of the dam as possible indicators for early selection of llamas for meat production. In the young, the development of hips and thighs at 2, 8, and 15 months of age and the correlation of these measurements with body weight and chest circumference were evaluated, whereas in the dams, the relation between size of the mammary gland and body weight at weaning of the calf was evaluated. Size of hip was calculated as a geometric figure using the Autocad software, and the size of the thigh was expressed as the volume of a truncated cone. The increase of body weight (65.3\%), hip area (51.5\%), thigh volume $(53.2 \%)$ were significantly higher $(\mathrm{p}<0.05)$ than the increase in chest circumference $(32.9 \%)$. Animals were distributed into two groups according to hip area at 2 months of age and the differences in biometric measurements were kept at 8 and 15 months of age. These differences were also kept in the other variables (thigh volume, chest circumference and body weight), indicating that body development was similar in both groups. Correlation coefficients between hip area, thigh volume, chest circumference, body weight and age were significant $(\mathrm{p}<0.001)$. Coefficient correlations between udder measurements and body weight at weaning were small, with the exception of distance among anterior udder teats and body weight at weaning (0.46). Results suggested that hip area and thigh volume can be used as genetic indicators for early selection of young animals for meat production as they keep a high and significant correlation with body weight and chest circumference.

Key words: hip, thigh, mammary gland, phenotypic indicators, development rate

\section{INTRODUCCIÓN}

La política actual del sector agrícola estatal, en lo concerniente a la producción de camélidos sudamericanos, prioriza la producción de carne para la satisfacción de la demanda de un mercado local exigente en un tipo de carne de textura suave y salubre. Estas características pueden lograrse en carcasas provenientes de animales jóvenes, cuyos padres fueron seleccionados para la producción de carne.

El rendimiento de carcasa de la llama es del 58\% (Bravo et al., 1981), frente al 53\% de la alpaca (Calderón y FernándezBaca, 1972), por lo que se considera que es la especie de la familia de los camélidos sudamericanos con el mayor potencial para la producción de carne; sin embargo, bajo las condiciones actuales de crianza, la carne de estos animales proviene mayormente de animales adultos, que asociado a una alimentación basada exclusivamente en pastura nati- va de bajo nivel nutricional (Leyva, 1991), producen carcasas de bajo rendimiento y calidad, lo cual afecta su valor y aceptación en el mercado.

Al presente, no existe un programa de mejoramiento genético relevante para la producción de carne de llama ni núcleos de reproductores que facilite la selección de estos animales. La selección debe hacerse a una edad temprana, en base a indicadores genéticos que expresen la capacidad genética en la tasa de crecimiento de la progenie. Así, el aporte genético de los padres ha sido relacionado con el peso corporal al nacimiento, destete y año de edad de la progenie en rumiantes (Legates y Warwick, 1992; Falconer y Trudy, 1996) y en alpacas y llamas (Sirna, 1984) para predecir el rendimiento de la carcasa.

Un aporte importante de la expresión genética del peso al destete de la cría proviene de la habilidad materna, expresado en la capacidad secretoria de leche de la glándula 
mamaria, lo cual, también, está ligado a un control genético (Miller et al., 1999). Es posible que esto mismo ocurra en llamas, basado en la mayor ganancia de peso de crías de alpacas con amamantamiento hasta los 10 meses en comparación con crías destetadas (Leyva, 1991); sin embargo, en la llama se desconoce la relación entre el tamaño de la glándula mamaria con la producción de leche y la tasa de crecimiento de la cría al destete y año de edad.

La producción de carne de un animal es evaluado a través del rendimiento de su carcasa, el cual es la expresión del desarrollo de su masa muscular (Rice et al., 1970), y se espera que las medidas corporales anatómicas que la expresen podrían ser los indicadores apropiados para seleccionar llamas jóvenes para producción de carne. En ovinos de carne, la zona crural representa el $35 \%$ del peso corporal, donde el área del muslo y la grupa aportan la mayor masa muscular. Basado en estas informaciones, el presente estudio tiene el propósito de evaluar el área de la grupa y el volumen del muslo de la cría, así como el tamaño de la glándula mamaria, como posibles indicadores para la selección temprana de llamas para la producción de carne.

\section{Materiales y Métodos}

El estudio se realizó en la Estación Experimental del Centro de Investigación IVITA, Facultad de Medicina Veterinaria, Universidad Nacional Mayor de San Marcos, localizada en el distrito de Maranganí, provincia de Canchis, departamento de Cusco. El área de crianza de las llamas se encontraba a una altitud aproximada de 4,200 msnm.

Se utilizó un rebaño de 82 llamas madres con sus respectivas crías. En las madres se midió el área de la ubre y en las crías, el volumen del muslo, el área de la grupa, el perímetro torácico y el peso corporal. La edad, número de parto y grupo racial de las madres se obtuvo de los registros del centro experi- mental. Todos los animales estuvieron expuestos a las mismas condiciones de manejo, sanidad y alimentación.

El volumen del muslo fue determinado por la circunferencia de los perímetros superior e inferior y la longitud del muslo. Se tomó como punto de referencia la altura de la inserción del pliegue de la babilla y la línea inguinal para la medida del perímetro superior, y la articulación fémoro-tibio-rotuliana para el perímetro inferior. La longitud se midió en la cara medial interna del muslo desde el punto medio de la línea inguinal hasta el punto medio de la articulación fémoro-tibio-rotuliana. La relación de ambas circunferencias y la longitud del muslo determinó la figura geométrica de un cono truncado (Fig. 1), cuyo volumen se calculó con la fórmula correspondiente.

Para el tamaño de la grupa se tomaron como referencias anatómicas las tuberosidades isquiática medial (A), sacra (B), coxal (C) y la isquiática lateral (D), midiéndose la distancia en forma secuencial (A-B, B-C, C$\mathrm{D}, \mathrm{D}-\mathrm{A})$. El área de la figura geométrica formada por la unión de los 4 puntos (Fig. 1) fue calculada con el programa Autocad 2004.

Se determinó el área de la grupa de las crías a los 2 meses de edad, y tomando como límite el promedio se estableció un grupo de crías con grupa pequeña y otro con grupa grande para evaluar si esta diferencia se encuentra relacionada con el volumen de muslo, peso corporal y perímetro torácico a los 2, 8 y 15 meses de edad. Así mismo, se estimó el incremento mensual a fin de predecir la ganancia de peso esperado debido al efecto genético para alcanzar el peso corporal adecuado para su comercialización.

El tamaño de la ubre de las madres se determinó a los 15 días post destete. Se usó la base de implantación como punto de referencia para medir la línea longitudinal que separa los cuartos derechos de los izquierdos, y la transversal que separa los cuartos anteriores de los posteriores. Además, se 


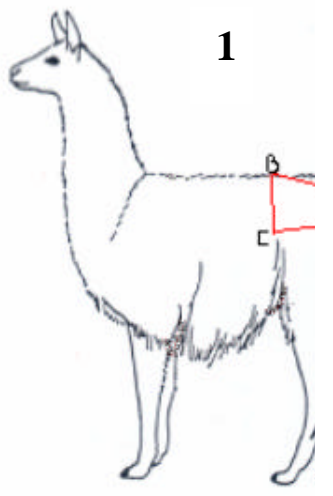

2

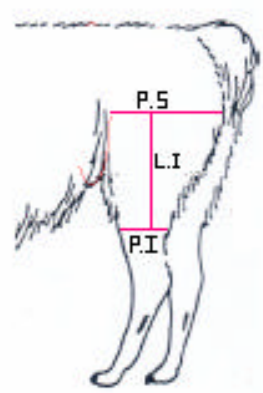

3

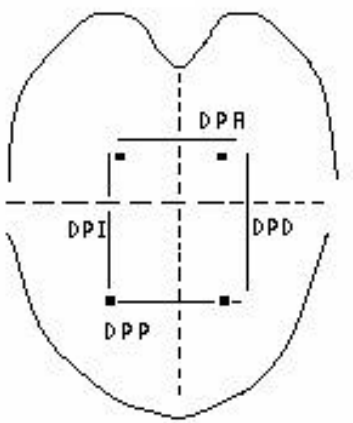

Figura 1. Determinación del área de la grupa (1), del volumen del muslo (2) y del tamaño de la ubre (3). A: Tuberosidad isquiática medial, B: Tuberosidad sacra, C: Tuberosidad coxal, D: Tuberosidad isquiática lateral, PS: Perímetro superior, PI: Perímetro inferior, LI: Línea interna, DPA: Distancia de pezones anteriores, DPP: Distancia de pezones posteriores, DPD: Distancia de pezones derechos, DPI: Distancia de pezones izquierdos.

determinó la distancia del intervalo transversal y longitudinal entre pezones. El área de la ubre fue determinada con las medidas longitudinal y transversal y en base al promedio y desviación estándar se estableció dos grupos (ubre grande y pequeña) y se les relacionó con el peso de las crías al destete ( 8 meses).

Para el registro de perímetro torácico, se tomaron como puntos de referencia la parte anterior del tórax (inmediatamente detrás del área axilar) y la cruz (encuentro de los omóplatos), utilizándose una cinta volumétrica de $1.5 \mathrm{~m}$ de longitud. El peso corporal fue registrado de 06:00 a 08:00 con los animales en ayunas. El peso al nacimiento se midió con una balanza romana $( \pm 25 \mathrm{~g})$ y para el peso corporal a los 2, 8 y 15 meses de edad con una balanza de reloj con una precisión de $0.025 \mathrm{~kg}$.

Se usó el análisis de la variancia (ANOVA), del paquete estadístico de SAS (SAS, 1993) para analizar el tamaño de la grupa y del muslo a los 2, 8 y 15 meses de edad.
La relación entre el área de la grupa y el volumen del muslo con el peso corporal y perímetro torácico se estableció a través de la correlación de Pearson (SAS, 1993). Los cambios en el tamaño de grupa y muslo con el crecimiento de la cría a los 2, 8 y 15 meses se estimaron mediante el análisis de regresión simple.

Se ajustó los datos de las mediciones zoométricas a las edades de 8 y 15 meses de edad (Legates y Warwick, 1992). No se ajustó a la edad de 2 meses ya que las mediciones se hicieron a la edad exacta.

\section{Resultados}

El incremento porcentual desde los 2 hasta los 15 meses de edad fue significativamente mayor $(\mathrm{p}<0.05)$ en el peso corporal $(65.3 \%)$, área de la grupa $(51.5 \%)$ y volumen del muslo (53.2\%) que en el perímetro torácico $(32.9 \%)$. Este mismo patrón se observó al analizar los datos en los periodos de 2 a 8 meses y de 8 a 15 meses de edad (Cuadro 1). 
Cuadro 1. Área de la grupa, volumen del muslo, perímetro torácico y peso corporal durante el crecimiento de crías de llamas (promedio \pm desviación estándar)

\begin{tabular}{lccc}
\hline Variable & 2 meses & 8 meses & 15 meses \\
\hline Área de la grupa $\left(\mathrm{cm}^{2}\right)$ & $89.3 \pm 12.3^{\mathrm{a}}$ & $146.5 \pm 22.7^{\mathrm{b}}$ & $184.3 \pm 23.7^{\mathrm{c}}$ \\
Volumen del muslo $\left(\mathrm{cm}^{3}\right)$ & $1,693.6 \pm 322.8^{\mathrm{a}}$ & $2,157.1 \pm 470.0^{\mathrm{b}}$ & $3,617.7 \pm 611.3^{\mathrm{c}}$ \\
Perímetro torácico $(\mathrm{cm})$ & $64.4 \pm 4.7^{\mathrm{a}}$ & $84.9 \pm 4.5^{\mathrm{b}}$ & $95.9 \pm 5.6^{\mathrm{c}}$ \\
Peso corporal $(\mathrm{kg})$ & $22.0 \pm 3.8^{\mathrm{a}}$ & $39.3 \pm 4.7^{\mathrm{b}}$ & $63.5 \pm 8.9^{\mathrm{c}}$ \\
\hline
\end{tabular}

${ }^{a, b, c}$ Valores con letras diferentes dentro de filas son significativamente diferentes $(p<0.05)$

Cuadro 2. Área de la grupa, volumen de muslo, peso corporal y perímetro torácico durante el crecimiento de crías de llamas (promedio \pm desviación estándar) de llamas distribuidas en dos grupos según el área de grupa que presentaron a los dos meses de edad

\begin{tabular}{|c|c|c|c|c|c|c|c|c|}
\hline \multirow{2}{*}{ Edad } & \multicolumn{2}{|c|}{$\begin{array}{l}\text { Área de la grupa } \\
\qquad\left(\mathrm{cm}^{2}\right)\end{array}$} & \multicolumn{2}{|c|}{$\begin{array}{l}\text { Volumen del muslo } \\
\qquad\left(\mathrm{cm}^{3}\right)\end{array}$} & \multicolumn{2}{|c|}{$\begin{array}{l}\text { Peso corporal } \\
(\mathrm{kg})\end{array}$} & \multicolumn{2}{|c|}{$\begin{array}{l}\text { Perímetro torácico } \\
\qquad(\mathrm{cm})\end{array}$} \\
\hline & Mayor & Menor & Mayor & Menor & Mayor & Menor & Mayor & Menor \\
\hline 2 meses & $98 \pm 9^{a}$ & $79 \pm 6^{b}$ & $1811 \pm 276^{\mathrm{a}}$ & $1559 \pm 323^{b}$ & $24 \pm 3^{\mathrm{a}}$ & $20 \pm 4^{b}$ & $66 \pm 4^{a}$ & $63 \pm 5^{b}$ \\
\hline 8 meses & $153 \pm 21^{\mathrm{a}}$ & $139 \pm 21^{b}$ & $2318 \pm 463^{\mathrm{a}}$ & $1974 \pm 414^{\mathrm{b}}$ & $42 \pm 4^{\mathrm{a}}$ & $37 \pm 4^{\mathrm{b}}$ & $86 \pm 4^{\mathrm{a}}$ & $83 \pm 5^{b}$ \\
\hline 15 meses & $190 \pm 23^{\mathrm{a}}$ & $178 \pm 23^{b}$ & $3805 \pm 641^{\mathrm{a}}$ & $3405 \pm 505^{\mathrm{b}}$ & $66 \pm 9^{a}$ & $61 \pm 9^{b}$ & $97 \pm 6^{\mathrm{a}}$ & $95 \pm 5^{b}$ \\
\hline
\end{tabular}

${ }^{a, b}$ Valores con letras diferentes dentro de filas y por variable son significativamente diferentes $(p<0.05)$

Las diferencias establecidas en la clasificación por tamaño de la grupa a los dos meses de edad se mantuvieron en las mediciones realizadas a los 8 y 15 meses de edad $(\mathrm{p}<0.05)$, y estas diferencias se mantuvieron en las otras variables bajo estudio (volumen de muslo, perímetro torácico y peso corporal), lo cual indica que la tasa de incremento fue similar en ambos grupos (Cuadro 2).

Las ecuaciones de regresión (Cuadro 3) muestran que el desarrollo mensual del área de la grupa y del perímetro torácico fue mayor en las crías 2 a 8 meses que en aquellas de 8 a 15 meses de edad $\left(9.5\right.$ vs $7.9 \mathrm{~cm}^{2}$ y 3.4 vs $2.3 \mathrm{~cm}$, respectivamente), mientras que el incremento mensual del volumen del mus- lo y el peso corporal fue mayor en las crías de 8 a 15 meses que en aquellas de 2 a 8 meses de edad (304.0 vs $77.3 \mathrm{~cm}^{3}$ y 5.0 vs. $2.9 \mathrm{~kg}$, respectivamente).

Los Cuadros 4 y 5 muestran los coeficientes de correlación entre el área de la grupa, volumen del muslo, peso corporal, perímetro torácico y edad desde los 2 a los 15 meses de edad. Los coeficientes de correlación fueron altos y significativos $(\mathrm{p}<0.001)$ entre estas variables (Cuadro 4). Similar patrón se observa en el análisis de las variables por tamaño de la grupa, excepto a los 2 meses de edad, donde las correlaciones en animales con grupa pequeña fueron medias o bajas (Cuadro 5). 
Cuadro 3. Ecuaciones de crecimiento del área de la grupa, volumen del muslo, perímetro torácico y peso corporal en llamas de 2 a 8 meses y de 8 a 15 meses

\begin{tabular}{lll}
\hline Variable & \multicolumn{1}{c}{2 a 8 meses } & 8 a 15 meses \\
\hline Área de la grupa $\left(\mathrm{cm}^{2}\right)$ & $\mathrm{y}=70.20+9.52 \mathrm{x}$ & $\mathrm{y}=83.5+7.87 \mathrm{x}$ \\
Volumen del muslo $\left(\mathrm{cm}^{3}\right)$ & $\mathrm{y}=1539+77.28 \mathrm{x}$ & $\mathrm{y}=-276+304 \mathrm{x}$ \\
Perímetro torácico $(\mathrm{cm})$ & $\mathrm{y}=57.44+3.43 \mathrm{x}$ & $\mathrm{y}=66.64+2.29 \mathrm{x}$ \\
Peso corporal $(\mathrm{kg})$ & $\mathrm{y}=16.24+2.86 \mathrm{x}$ & $\mathrm{y}=-0.93+5.03 \mathrm{x}$ \\
\hline
\end{tabular}

Cuadro 4. Coeficientes de correlación entre el área de la grupa, volumen del muslo, peso corporal, perímetro torácico y edad en llamas de 2 a 15 meses de edad

\begin{tabular}{lccccc}
\hline Variable & $\begin{array}{c}\text { Área de la } \\
\text { grupa }\end{array}$ & $\begin{array}{c}\text { Perímetro } \\
\text { torácico }\end{array}$ & $\begin{array}{c}\text { Peso } \\
\text { corporal }\end{array}$ & $\begin{array}{c}\text { Volumen } \\
\text { del muslo }\end{array}$ & Edad \\
\hline Área de la grupa & & 0.93 & 0.93 & 0.85 & 0.89 \\
Perímetro torácico & 0.93 & & 0.94 & 0.84 & 0.93 \\
Peso corporal & 0.92 & 0.94 & & 0.91 & 0.92 \\
Volumen del muslo & 0.85 & 0.85 & 0.91 & & 0.79 \\
Edad & 0.89 & 0.93 & 0.92 & 0.79 & \\
\hline
\end{tabular}

Cuadro 5. Coeficientes de correlación del área de grupa grande y pequeña con el volumen de muslo, peso corporal y perímetro torácico de llamas a los 2, 8 y 15 meses de edad

\begin{tabular}{llccc}
\hline \multirow{2}{*}{ Variable } & Grupa & \multicolumn{3}{c}{ Área de la grupa } \\
\cline { 3 - 5 } & & 2 meses & 8 meses & 15 meses \\
\hline \multirow{2}{*}{ Volumen del muslo } & Grande & 0.62 & 0.62 & 0.73 \\
& Pequeño & 0.45 & 0.57 & 0.63 \\
\multirow{2}{*}{ Peso corporal } & Grande & 0.58 & 0.64 & 0.72 \\
& Pequeño & 0.07 & 0.77 & 0.73 \\
\multirow{2}{*}{ Perímetro torácico } & Grande & 0.56 & 0.59 & 0.64 \\
& Pequeño & 0.14 & 0.71 & 0.62 \\
\hline
\end{tabular}


Cuadro 6. Regresión lineal entre (a) el área de la grupa y el peso de la cría, y (b) el peso y el área de la grupa de la cría de llama a los 2, 8 y 15 meses de edad

\begin{tabular}{ccccc}
\hline $\begin{array}{c}\text { Intercepto } \\
\text { estimado } \pm \text { e.e. }\end{array}$ & $\begin{array}{c}\text { Observaciones } \\
(\mathrm{n})\end{array}$ & Regresor & $\begin{array}{c}\text { Coeficiente } \\
\text { estimado }\end{array}$ & $\mathrm{R}^{2}$ \\
\hline Área de la grupa y el peso de la cría & & & & \\
$-11.79 \pm 1.66$ & 192 & 2 a $15 \mathrm{~m}$ & $0.38 \pm 0.01$ & 0.86 \\
$-0.07 \pm 1.24$ & 127 & 2 a $8 \mathrm{~m}$ & $0.26 \pm 0.01$ & 0.84 \\
$-12.68 \pm 3.97$ & 127 & 8 a $15 \mathrm{~m}$ & $0.38 \pm 0.02$ & 0.68 \\
Peso de la cría y el área de la grupa & & & & \\
$46.60 \pm 3.02$ & 192 & 2 a $15 \mathrm{~m}$ & $2.24 \pm 2.06$ & 0.85 \\
$19.20 \pm 4.03$ & 127 & $2 \mathrm{a} 8 \mathrm{~m}$ & $3.21 \pm 0.12$ & 0.83 \\
$75.06 \pm 5.70$ & 127 & 8 a $15 \mathrm{~m}$ & $1.75 \pm 0.10$ & 0.68 \\
\hline
\end{tabular}

Cuadro 7. Coeficientes de correlación de las medidas biométricas de la ubre de la madre con el peso de la cría al destete

\begin{tabular}{|c|c|c|c|c|c|c|c|}
\hline & L. ubre & A. ubre & DEPA & DEPP & DEPD & DEPI & Peso \\
\hline Área & $\begin{array}{c}0.78 \\
(0.01)\end{array}$ & $\begin{array}{c}0.92 \\
(0.01)\end{array}$ & $\begin{array}{c}0.11 \\
(0.37)\end{array}$ & $\begin{array}{c}-0.02 \\
(0.87)\end{array}$ & $\begin{array}{c}0.27 \\
(0.03)\end{array}$ & $\begin{array}{c}0.34 \\
(0.01)\end{array}$ & $\begin{array}{c}0.28 \\
(0.02)\end{array}$ \\
\hline L. ubre & & $\begin{array}{c}0.52 \\
(0.01)\end{array}$ & $\begin{array}{c}-0.3 \\
(0.78)\end{array}$ & $\begin{array}{c}-0.02 \\
(0.86)\end{array}$ & $\begin{array}{c}0.21 \\
(0.10)\end{array}$ & $\begin{array}{c}0.28 \\
(0.02)\end{array}$ & $\begin{array}{c}0.27 \\
(0.03)\end{array}$ \\
\hline A. ubre & & & $\begin{array}{c}0.18 \\
(0.16)\end{array}$ & $\begin{array}{c}-0.02 \\
(0.86)\end{array}$ & $\begin{array}{c}0.20 \\
(0.11)\end{array}$ & $\begin{array}{c}0.23 \\
(0.06)\end{array}$ & $\begin{array}{c}0.21 \\
(0.09)\end{array}$ \\
\hline DEPA & & & & $\begin{array}{c}0.46 \\
(0.01)\end{array}$ & $\begin{array}{l}-0.10 \\
(0.42)\end{array}$ & $\begin{array}{l}-0.07 \\
(0.56)\end{array}$ & $\begin{array}{r}0.46 \\
(0.01\end{array}$ \\
\hline DEPP & & & & & $\begin{array}{l}-0.10 \\
(0.42)\end{array}$ & $\begin{array}{l}-0.05 \\
(0.68)\end{array}$ & $\begin{array}{c}0.05 \\
(0.68)\end{array}$ \\
\hline DEPD & & & & & & $\begin{array}{c}0.90 \\
(0.01)\end{array}$ & $\begin{array}{r}-0.012 \\
(0.92)\end{array}$ \\
\hline Peso & & & & & & & $\begin{array}{c}0.04 \\
(0.74)\end{array}$ \\
\hline
\end{tabular}

Área: Área de ubre; L. ubre: Largo de ubre; A. ubre: Ancho de ubre; DEPA: distancia entre pezones anteriores; DEPP: distancia entre pezones posteriores; DEPD: distancia entre pezones derechos; DEPI: distancia entre pezones izquierdos; Peso: peso corporal de la cría al destete

( ) Nivel de significancia 
Las ecuaciones de predicción (Cuadro 6) para estimar el peso a partir del área de la grupa, muestran que por cada $\mathrm{cm}^{2}$ de incremento del área de la grupa en las crías de $2 \mathrm{a}$ 15,2 a 8 y 8 a 15 meses de edad se incrementa el peso en $0.38,0.26$ y $0.38 \mathrm{~kg}$, respectivamente. A la inversa, la regresión del área de la grupa en función del peso corporal en la cría sugiere que por cada incremento de $1 \mathrm{~kg}$ de peso vivo, el área de la grupa incrementa en $2.24,3.21$ y $1.75 \mathrm{~cm}^{2}$ en las crías de 2 a 15,2 a 8 y de 8 a 15 meses de edad, respectivamente.

El Cuadro 7 muestra las relaciones entre la medida de la ubre y el peso de la cría al destete. La relación más alta es la del ancho de la ubre con el área de la ubre (0.92). No se observó asociaciones significativas con las distancias entre pezones izquierdos, derechos y posteriores. Al relacionar las medidas de la ubre con el peso de la cría al destete se hallaron coeficientes bajos a medios como distancia entre pezones anteriores (0.46), seguida del largo de ubre (0.27) y del área de la ubre (0.28).

\section{Discusión}

El crecimiento y desarrollo corporal es evaluado a través del cambio en el peso corporal, lo cual incluye las vísceras, piel, masa muscular y hueso; sin embargo, la condición cárnica de un animal es estimada con mayor precisión evaluando la condición corporal (O'Mara et al., 1998). En vacunos de carne se usa el área de la grupa como punto de referencia para la condición corporal (Green, et al., 1972; O'Mara et al., 1998) y el desarrollo de la masa muscular del muslo (O'Mara et al., 1998). En el presente trabajo se estudió estas dos variables como indicadores para estimar el efecto genético en el crecimiento y la ganancia de peso para la selección temprana de llamas para producción de carne. Los resultados sugieren que las variables área de la grupa y volumen del muslo, al guardar una relación alta, positiva y significativa con el peso corporal y perímetro torácico, podrían ser utilizados como indicadores para la selección temprana de animales con mayor potencial de desarrollo.

La implicancia genética se refuerza por los resultados obtenidos al comparar grupos de crías similares en edad, pero con mayor y menor desarrollo del área de la grupa, donde se mantienen las diferencias entre grupos a medida que la cría incrementa en edad; sin embargo, es necesario ratificar el efecto genético y descartar el efecto compensatorio en la diferencia en el desarrollo dentro de estas dos variables, si los animales fueran expuestos a un nivel alto de alimentación.

Por otro lado, el patrón de desarrollo del peso corporal y perímetro torácico de los animales clasificados por mayor y menor medida fue similar al desarrollo del área de la grupa y volumen del muslo de la misma clase, demostrado por la correlación positiva y altamente significativa encontradas entre estas variables. Estos resultados indican que las variables área de la grupa y volumen del muslo son indicadores relevantes de la condición del peso corporal y del perímetro torácico. Otros estudios que apoyan la hipótesis del área de la grupa, como un indicador en la selección de animales para producción de carne, son los de Estrada (1983), con correlaciones de 0.77 y 0.81 entre largo de la grupa y peso corporal, para llamas machos tuis y adultos, respectivamente, y los de Bustinza (1993), con correlaciones de 0.58 y 0.52 entre peso corporal y distancia entre las puntas de la cadera para alpacas criadas en pastos cultivados y nativos, respectivamente. Es probable que las correlaciones obtenidas en llamas hayan sido más altas debido a las características de una cadera asociada a un desarrollo cárnico a diferencia de la alpaca con orientación en la producción de fibra.

El estudio demuestra que se puede predecir con bastante exactitud el incremento de peso corporal en base al incremento mensual del área de la grupa $\left(\mathrm{R}^{2}=0.84\right)$, así como la predicción del incremento del área de la 
grupa en base al incremento mensual del peso corporal $\left(\mathrm{R}^{2}=0.85\right)$ para los periodos de $2 \mathrm{a}$ 15 meses de edad. Esta información podría ser utilizada en la predicción del rendimiento de carcasa y la edad de beneficio apropiado. Hammack y Shrode (1986) señalan que el peso y algunas medidas corporales como la longitud corporal y el ancho de caderas son útiles para predecir los pesos de carcasa en vacunos de carne; así mismo, Yao et al. (1953) encontraron en bovinos de carne que el diámetro y circunferencia muscular estaban positivamente correlacionadas con el grado de la canal. En base a estas informaciones, se puede inferir que el área de la grupa y el volumen del muslo deben guardar estrecha relación con el rendimiento de la carcasa, ya que son zonas de gran desarrollo muscular, hecho que debe corroborarse en la llama.

En otros estudios sobre peso corporal en llamas, se encontró que el perímetro torácico fue la variable más apropiada para predicción del peso corporal $(\mathrm{r}=0.91$; Wurzinger, 2003), y este coeficiente de correlación fue similar al encontrado en el presente estudio entre peso corporal y área de grupa (0.92) y volumen de muslo (0.91) y entre perímetro torácico y peso corporal (0.94). La alta correlación entre el área de la grupa con el peso corporal responde al hecho de que la medida de la grupa involucra medidas de los huesos íleon, isquion y pubis, los cuales guardan una relación más directa con la tasa de desarrollo en animales en crecimiento (McMeeckan, 1960).

Los resultados muestran un desarrollo más temprano en el área de la grupa (formado por huesos pélvicos) en el periodo de 2 a 8 meses, a diferencia de un desarrollo más pronunciado del muslo en el periodo de 8 a 15 meses. Esto se explica por el hecho fisiológico de que el músculo en crías en crecimiento presenta un desarrollo más tardío que el hueso, y a medida que las crías llegan a la madurez, los aumentos de peso involucran cantidades crecientes de grasa (McDonald et al., 1995).
El crecimiento de las crías durante el primer mes de vida depende de la producción de leche de la madre (Leyva et al., 1983; Jiménez, 1984) y, además, en una fase posterior del crecimiento, la leche juega un rol adicional muy importante para el desarrollo de la alpaca y la llama (Leyva, 1983; Leyva y Markas, 1991). Por otro lado, el mayor volumen de producción de leche puede depender del desarrollo o tamaño de la glándula mamaria, tal como ocurre en bovinos de carne (Miller et al., 1999); sin embargo, en el presente estudio se encontró que la correlación entre el tamaño de la glándula mamaria y el peso de las crías al destete fueron medias y bajas. Es probable que el registro del tamaño de las glándulas mamarias, alrededor de los 15 días después del destete, en un estado de involución completa, no haya sido el momento más apropiado para evidenciar el potencial en la producción de leche, por cuanto el desarrollo de la glándula mamaria y la producción de leche están gobernados hormonalmente, eventos que ocurren cuando la madre se encuentra en lactación. Gonzáles (2006) encontró una correlación positiva y significativamente alta entre producción de leche y tasa de crecimiento de crías de madres llamas seleccionadas para producción de carne; así mismo, Quispe (1973) encontró una correlación fenotípica significativa entre medidas de la ubre con la producción láctea en vacas.

Por último, el hallazgo de una correlación positiva, media y significativa entre distancia entre pezones anteriores y peso de la cría al destete, sugiere que esta medida podría expresar la capacidad láctea de la glándula mamaria, información que requiere ser confirmada a través de un diseño experimental específico.

\section{Conclusiones}

- El área de la grupa y el volumen del muslo pueden ser usados como indicadores para la selección temprana de llamas para producción de carne. 
- Los puntos anatómicos de referencias para estas medidas son factibles de ser registrados por el productor.

\section{Literatura Citada}

1. Bravo, W.; E. Franco; J. Sumar. 1981. Rendimiento de canal en llamas. IV Conv. Internac. sobre Camélidos Sudamericanos. Punta Arenas, Chile.

2. Bustinza, V. 1993. Carne de alpaca. Escuela de Postgrado, Universidad Nacional del Altiplano. Puno Perú. 140 p.

3. Calderón, W.; S. Fernández-Baca. 1972. Peso vivo y rendimiento de canal de alpaca. Rev. Inv. Pec. IVITA 1(1): 5-9.

4. Estrada, A. 1983. Zoometría y algunas correlaciones en llamas. Tesis de Ingeniero Zootecnista. Facultad de Zootecnia, Univ. San Antonio Abad del Cusco. Cusco. $81 \mathrm{p}$.

5. Falconer, D.; F. Trudy. 1996. Introducción a la genética cuantitativa. Ed. Acribia. Zaragoza. España. 340 p.

6. Gonzáles, R. 2006. Efecto de dos niveles nutricionales sobre la producción de leche en llamas seleccionadas para producción de carne. Tesis de Médico Veterinario. Facultad de Medicina Veterinaria, Univ. Nacional Mayor de San Marcos. Lima. 52 p.

7. Green, W.; W. Stevens; M. Gauch. 1972. Use of body measurements to predict the weigths of whosale cuts of beef carcasses combination cuts of 900 pound steers. Bulletin A-180. Agriculture Experiment Station, College Park. Maryland, USA. 14 p.

8. Hammack, S.; R. Shrode. 1986. Calfhood weights, body measurements and measures of fatness versus criteria of overall size and shape for predicting yearling performance in beef cattle. J. Anim. Sci. 63: 447-452.

9. Jiménez, J. 1984. Efecto de la edad sobre la producción de leche de alpacas bajo condiciones de pastura natural. Te- sis de Medico Veterinario. Facultad de Medicina Veterinaria, Univ. Nacional Mayor de San Marcos. Lima. 32 p.

10. Legates, J.; J. Warwick. 1992. Cría y mejora del ganado. $8^{\mathrm{a}}$ ed. Interamericana McGraw-Hill. Atlampa, México. 344 p.

11. Leyva, V. 1991. Camélidos Sudamericanos. Informe Técnico Fase 3. IVITA CIID-Canadá. Lima. 89 p.

12. Leyva, V.; E. Franco; N. Condorena. 1983. Determinación de la curva lactacional en alpacas y llamas en condiciones de pastura natural. VI Reunión Asoc. Per. Prod. Anim. Lambayeque, Perú.

13. Leyva, V.; J. Markas. 1991. Involución de la glándula mamaria en alpacas y efecto sobre el peso corporal y producción de fibra. Turrialba (IICA) 41(1): 59-63.

14. McDonald, P.; R. Edwards; J. Greenghalg; C. Morgan. 1995. Nutrición animal. Ed. Acribia. Zaragoza, España. 576 p.

15. McMeeckan, C.P. 1960. Principles of animal production. Ed. Whitcomb/ Tombs. Canterbury, New Zealand. 319 p.

16. Miller, S.; J. Wilton; W. Pfeiffer. 1999. Effects ok milk yield on biological efficiency and profit of beef production from birth to slaughter. J. Anim. Sci. 77: 344-352.

17. O'Mara, F.; S. Williams; J. Tatum; G. Hilton; T. Pringle; J. Wise; F. Williams. 1998. Prediction of slaughter cow composition using live animal and carcass traits. J. Anim. Sci. 76: 15941603.

18. Quispe, J. 1973. Correlación entre medidas biométricas de la ubre y la producción de leche en vacas Holstein. Tesis de Ingeniero Zootecnista. Facultad de Zootecnia, Univ. Nacional Agraria La Molina. Lima. 72 p.

19. Rice, V.; F. Andrews; F. Warwick; J. Legates. 1970. Improving beef cattle. In: Breeding and improvements of farm animals. p 309-355. Ed. McGraw-Hill. USA. 
20. SAS. 1993. SAS/STAT. User's Guide, version $6.4^{\text {th }}$ ed. Vol 1. SAS Institute. Cary, NC, USA. 943 p.

21. Sirna, C. 1984. Índices productivos de alpacas gestantes bajo dos sistemas de crianza en Puno. Tesis de Ingeniero Zootecnista. Facultad de Zootecnia, Univ. Nacional Agraria La Molina. Lima. 117 p.

22. Wurzinger, M.; J. Delgado; M. Nümberg; A. Valle; A. Stemmer; G.
Ugarte; J. Sölkner. 2003. Growth curves and genetic parameters for growth traits in Bolivian llamas. Livestock Prod. Sci. 95: 73-81.

23. Yao, T.S; W.M. Dawson; A.C. Cook. 1953. Relationship between meat production characters and body measurements in beef and milking shorthorn steers. J. Anim. Sci. 12: 775-786. 